Chirurg 2012 $\cdot 83: 822$

DOI 10.1007/s00104-012-2361-3

Online publiziert: 19. August 2012

(c) Springer-Verlag 2012

J. Reibetanz - C.T. Germer

Klinik für Allgemein-, Viszeral-, Gefäß- und Kinderchirurgie, Universitätsklinikum Würzburg

\title{
Exakte Beurteilung des zirkumferenziellen Resektionsrandes mittels MRT beim Rektumkarzinom
}

\section{Ergebnisse}

\section{Originalpublikation}

Al-Sukhni E, Milot L, Fruitman M et al (2012)

Diagnostic accuracy of MRI for assessment

of T category, lymph node metastases, and circumferential resection margin involvement in patients with rectal cancer: a systematic review and meta-analysis. Ann Surg Oncol 19:2212-2223

Ein exaktes prätherapetisches Lokalstaging hinsichtlich T-Kategorie, Nodalstatus und zirkumferenziellem Resektionsrand (CRM) sind entscheidende Selektionskriterien für die Einleitung einer neoadjuvanten Therapie beim Rektumkarzinom. Eine Unterschätzung des Tumorstadiums erhöht das Risiko einer inkompletten Resektion, während ein Overstaging eine unnötige Radio(Chemo)therapie indizieren kann.

\section{Fragestellung der Studie}

Das Ziel dieser systematischen Literaturrecherche war die Erhebung der diagnostischen Genauigkeit des prätherapeutischen MRT bezüglich der T-Kategorie (T1/2 vs. T3/4), des Nodalstatus und des CRM beim Rektumkarzinom.

\section{Methode}

Aus den bekannten medizinischen Datenbanken (Medline, EMBASE, Cochrane Database) wurden Publikationen zur Staginggenauigkeit des MRT beim Rektumkarzinom extrahiert. Das histopathologische Tumorstadium galt als Referenz. Es wurden ausschließlich Studien nach 2000 und Patienten ohne präoperative Radio(Chemo)therapie eingeschlossen.
Insgesamt 21 Studien wurden in die Metaanalyse eingeschlossen. Dabei handelte es sich um 8 retrospektive und 11 prospektive Analysen, bei 2 Studien war das Studiendesign unklar. Neunzehn Studien beinhalteten Ergebnisse zur T-Kategorie, 12 Studien zum Nodalstatus und 10 Studien zum CRM. Die eingeschlossenen Studien zeigten erwartungsgemäß eine relevante Heterogenität hinsichtlich der Definitionen eines positiven CRM $(\leq 5 \mathrm{~mm}$ bis direkte Involvierung der mesorektalen Faszie) und radiologisch positiver Lymphknoten (Größe, irreguläre Berandung, Signalverhalten). Die Ergebnisse bestätigten eine signifikant höhere Spezifität (richtig-negative Rate) des MRT in Bezug auf ein CRM-Involvement (94\%, 95\%-Konfidenzintervall [KI]: 88-97), verglichen mit der T-Kategorie (75\%, 95\%-KI: 68-80) bzw. dem Nodalstatus (71\%, 95\%-KI: 5981). Im Gegensatz dazu zeigten sich keine Unterschiede in der Sensitivität (richtig-positive Rate) der Methode in Bezug auf die drei Elemente. Höhere Feldstärken des MRT (3 T vs. 1,5 T) und übereinstimmende Zweitbefundung durch einen weiteren Radiologen erhöhten die Spezifität der Untersuchung hinsichtlich der T-Kategorie. Das MRT-Protokoll hatte dagegen keinen Einfluss auf die diagnostische Genauigkeit.

\section{Diskussion und Fazit des Reviewers}

Anhand der vorliegenden Metaanalyse konnte die hohe Spezifität (94\%) der präoperativen MRT in Bezug auf das Vor- liegen eines positiven CRM untermauert werden. Hinsichtlich der Parameter T-Kategorie und Nodalstatus zeigte die MRT eine signifikant geringere Spezifität. Diese „Ungenauigkeit“ kann jedoch, zumindest was die T-Kategorie anbelangt, durch höhere Feldstärken und eine Mitbegutachtung durch einen zweiten Radiologen gesteigert werden. Hauptproblem bei der Bewertung der diagnostischen Genauigkeit bleibt jedoch die uneinheitliche Definition radiologisch „positiver Fälle" bezüglich des jeweiligen Stagingparameters. Dies gilt insbesondere für den Lymphknotenstatus. Aber auch im Hinblick auf den CRM ist unklar, ab welchem radiologischen Cut-off ( $<$ vs. $\geq 5 \mathrm{~mm}$ ) bei adäquater Chirurgie auch histopathologisch von einem tumorfreien zirkumferenziellen Resektatrand $\geq 1 \mathrm{~mm}$ ausgegangen werden kann. Insgesamt unterstützen die Daten aber die besondere Wertigkeit der MRT als Staginguntersuchung beim Rektumkarzinom in Bezug auf die Indikationsstellung zur neoadjuvanten Therapie bzw. zur Abschätzung der Erreichbarkeit eines histopathologisch freien CRM.

\section{Korrespondenzadresse}

Prof. Dr. C.T. Germer

Klinik für Allgemein-, Viszeral-, Gefäß- und Kinderchirurgie, Universitätsklinikum Würzburg Oberdürrbacher Str. 6, 97080 Würzburg germer_c@klinik.uni-wuerzburg.de

Interessenkonflikt. Der korrespondierende Autor gibt für sich und seinen Koautor an, dass kein Interessenkonflikt besteht. 